\title{
Directivity of the sound radiated from a factory building
}

\author{
Gensei Matsumoto $^{1,2}$, Kyoji Fujiwara ${ }^{2}$ and Akira Omoto ${ }^{2}$ \\ ${ }^{1}$ Fukuoka Institute of Health and Environmental Sciences, \\ e-mail: g-matsumoto@fihes.pref.fukuoka.jp \\ ${ }^{2}$ Department of Acoustic Design, Kyushu Institute of Design
}

(Received 24 May 2001, Accepted for publication 13 July 2001)

Keywords: Directivity, Factory noise PACS number: 43.20.Fn, 43.50.Gf

\section{Introduction}

We investigated the effect of directivity of a sound source on the sound shielding efficiency of a noise barrier. It has been reported that the efficiency of shielding of a directional sound source having vertical directivity is less than the calculated value assuming an omni-directional sound source [1]. A source with vertical directivity might exist in a factory building. Sound radiation from the roof of the factory might be greater than that from the sidewall, because materials in a roof are usually lighter than those in a sidewall.

We propose here a numerical model of the directivities of the surroundings of a factory. We assumed the presence of a diffuse sound field inside the building and the radiated sound was evaluated using the geometrical theory of diffraction and the approximate expression for the potential of the multiple edge diffraction.

\section{Numerical model}

2.1. Sound radiation from element point sources

A three-dimensional sound filed is assumed. Figure 1 shows the building of a factory used for the calculation: the length parallel to the $z$-axis is infinite, the height of the sidewall is $5 \mathrm{~m}$, and the width of each roof is $5 \mathrm{~m}$ (inclination angle: $\pi / 6$ from the horizontal surface). Assuming a diffuse sound field, the acoustic energy density $E_{\text {in }}$ in the building is,

$$
E_{\text {in }}=\frac{4 W_{\text {in }}}{c A}\left(\mathrm{~W} \cdot \mathrm{s} / \mathrm{m}^{3}\right) \text {. }
$$

Where $W_{\text {in }}(\mathrm{W})$ is the sound power per unit of length parallel to the $z$-axis, $c(\mathrm{~m} / \mathrm{s})$ is the sound speed, and $A\left(\mathrm{~m}^{2}\right)$ is the sound absorption power per unit of length parallel to the $z$ axis. Consider that the element point sources are serially distributed on the roofs and the sidewalls, and that each point source which situates the center of the element point source having an area of $S\left(\mathrm{~m}^{2}\right)$ radiates sound incoherently. Then the sound power, $W_{\text {point }}$, radiated from each point source is described in the following.

$$
W_{\text {point }}=\frac{c}{4} E_{\mathrm{in}} S \tau(\mathrm{W})
$$

Where $\tau$ is a transmission coefficient. Therefore, the sound pressure level is obtained by adding the contributions of all point sources, for which the powers are shown in Eq. (2). The sound power level, $L_{\text {wall }}$, of an element point source situated on the sidewall and on the roof, $L_{\text {roof }}$, have a difference of

$$
L_{\text {roof }}-L_{\text {wall }}=10 \log _{10} \frac{1}{\tau_{\text {wall }}}-10 \log _{10} \frac{1}{\tau_{\text {roof }}} .
$$

Where $\tau_{\text {wall }}$ and $\tau_{\text {roof }}$ are transmission coefficients of the sidewall and the roof, respectively. The sound pressure level considering the contribution of all element point sources can be obtained by adding the value described in Eq. (3) on the occasion of energy-summation if the element point source is situated on the roof.

2.2. Calculation of sound pressure from a point source

The sound pressure from each point source is calculated using the approximate expression for the diffracted sound field around a many-sided pillar. Using a mirror image for the ground surface, consider that the building of the factory consists of six pillars, as shown in Fig. 2. By six cylindrical coordinates for the edge $i(i=1, \cdots, 6)$, source $S_{0}$ is described by $\left(r_{s_{i}}, \theta_{s_{i}}, z_{s}\right)$ and receiver $P_{0}$ by $\left(r_{i}, \theta_{i}, z\right)$. According to Keller's geometrical theory of diffraction [2], a sound field consists of a geometrical optics field and a diffracted field. The geometrical optics field, $\phi^{\mathrm{g}}$, is the sum of the fields on all incident and reflected rays, and the diffracted field, $\phi^{\mathrm{d}}$, is the sum of the fields on all diffracted rays. In the case of the pillar shown in Fig. 2, $\phi^{\mathrm{g}}$ is easily calculated because the aperture angle of each wedge is larger than $\pi$. As for $\phi^{\mathrm{d}}$, the potentials of rays that diffract the same ridgeline more than twice could be negligible. Furthermore, it is sufficient to calculate the potential for the single-edge, double-edge, and triple-edge diffraction, because the potential of a multiple diffracted ray that could pass from edge 4 through edge 5 and edge 6 to edge 3 is much less than that of a double-edge or triple-edge diffracted ray which would come around inversely. In order to obtain the potentials for the single-edge, double-edge, and triple-edge diffraction, we adopted Kawai's expressions [3], which expanded Kouyoumjian and Pathak's approximate expression.

The side length of a square that comprises an element point source is more than $\lambda / 2$ due to the limits of approximate expressions. Besides, the minimum that can divide the height of the sidewall and the width of the roof is selected as the side length of the square, because it might be agreeable that the element point sources are placed uniformly.

2.3. Comparison between measured and calculated values

To confirm that this numerical model adequately reproduces a sound field, we made measurements using a scale model. The factory building in Fig. 1 was reduced to a 


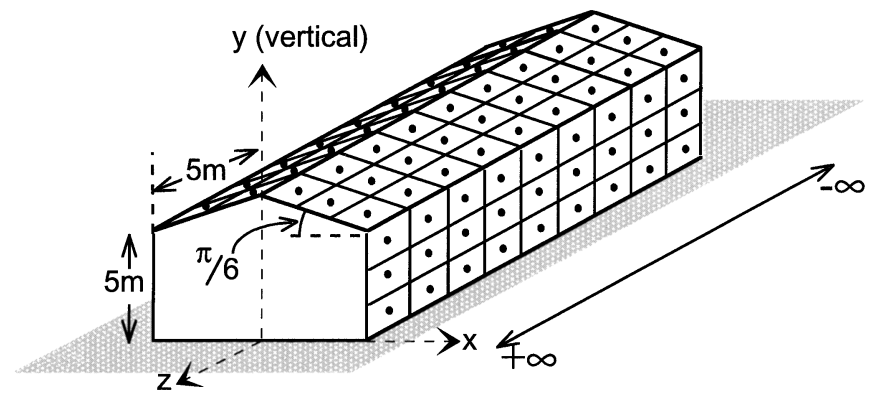

Fig. 1 Geometric size of the factory building.

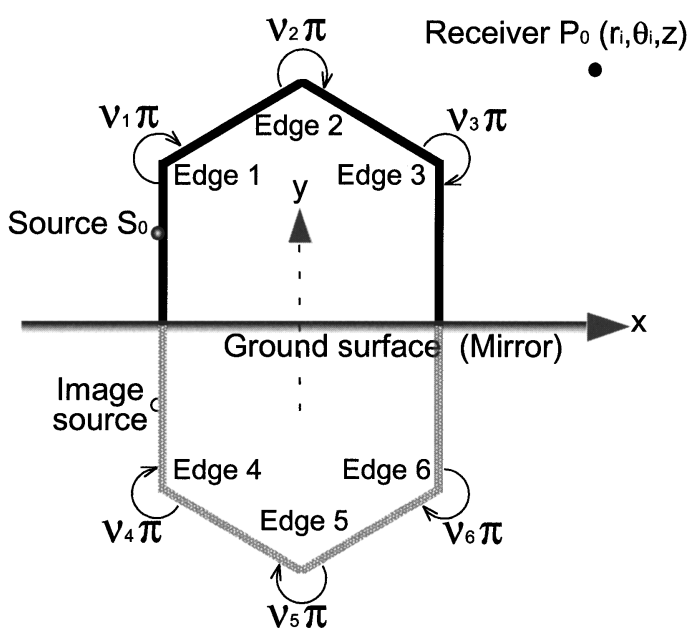

Fig. 2 Mirror image of the factory building.

$1 / 10$ scale model, and the length of the $z$-axis was cut down in $4 \mathrm{~m}$ because of the limit of the volume of the hemi-anechoic room. Both ends of the scale model were boarded up with chipped-wood board of $30 \mathrm{~mm}$ in thickness. In order to improve the diffuseness of the inner sound field, five speakers that radiate random noise were arranged and many curved iron plates were arranged as diffusers.

Acrylic boards were used for the sidewalls and roofs. The transmission loss of an acrylic board was calculated based on the mass law. The mass density is $1.19 \times 10^{3} \mathrm{~kg} / \mathrm{m}^{3}$. The surface for measurement was a cross-section perpendicular to the $z$-axis, and the sound pressure levels were measured on a semicircle, of which the center was $(x, y)=(0,0)$ and the radius was $10 \mathrm{~m}$ in actual size. Measurements were performed with an acrylic board of $1 \mathrm{~mm}$ in thickness for the roof and $2 \mathrm{~mm}$ in thickness for the sidewall, and also when these thicknesses for the sidewall and roof were reversed.

The patterns of directivity were also obtained by means of a numerical model considering the difference in transmission losses between the sidewall and the roof. Figure 3 shows a comparison between the measured and calculated values at $500 \mathrm{~Hz}$ and $1,000 \mathrm{~Hz}$. Even though the maximal gap is about $3 \mathrm{~dB}$, the fitting of the curve to the measurement value was good. Therefore, the numerical model shown above accurately reproduces a sound field around a factory building.

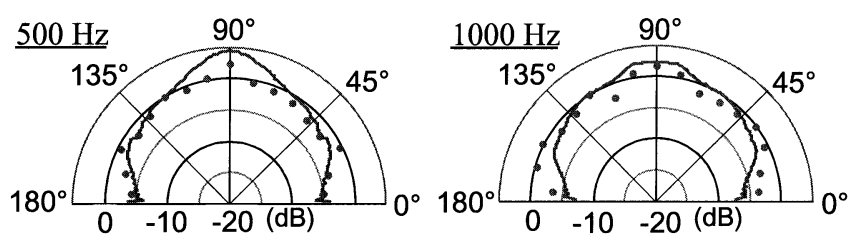

(a)
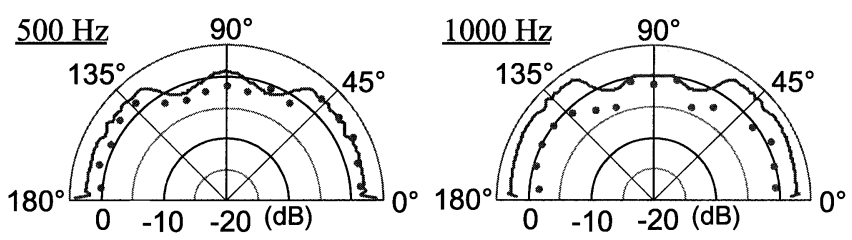

(b)

-: Measured value $\quad-$ : Calculated value

Fig. 3 Comparison between measured and calculated values on a semicircle having a radius of $10 \mathrm{~m}$ in actual size: (a) the roof and the sidewall are acrylic board of $1 \mathrm{~mm}$ in thickness and $2 \mathrm{~mm}$ in thickness, respectively, (b) the roof and the sidewall are acrylic board of $2 \mathrm{~mm}$ in thickness and $1 \mathrm{~mm}$ in thickness, respectively.

\section{Calculation of directivity}

Two cases of directivity were calculated when actual building materials were selected for the sidewall and the roof. In case 1, the sidewall was assumed to be sheet glass and concrete block $150 \mathrm{~mm}$ in thickness (the areal ratio was 1 to 4 ), and the roof was slate $6.5 \mathrm{~mm}$ in thickness. In case 2 , the sidewall was assumed to be the same as in case 1, but the roof was assumed to be composed of slates filled with light-weight concrete. The transmission loss in each case [4] is shown in Table 1.

Figure 4 shows the distributions of sound pressure levels around the building at $500 \mathrm{~Hz}$. In case 1, the center position of the sound radiation seems to be about $5 \mathrm{~m}$ above the ground. In this case, the transmission loss of the sidewall is $6.9 \mathrm{~dB}$ larger than that of the roof, so it might be considered that the contribution from the roof, which is larger than that from the sidewall, makes the source position higher. In case 2 , by contrast, the transmission loss of the sidewall is $4.1 \mathrm{~dB}$ smaller than that of the roof. Since the contribution from the sidewall is larger than that from the roof, the center position seems to be on the ground surface. Therefore the center position, considering radiation from all sidewalls and roofs, could move up and down according to the combination of transmission losses of the building materials. We suggest that

Table 1 Two cases of transmission losses for the sidewall and the roof.

\begin{tabular}{ccccc}
\hline & & $250 \mathrm{~Hz}$ & $500 \mathrm{~Hz}$ & $1,000 \mathrm{~Hz}$ \\
\hline \multirow{2}{*}{ Case 1 } & $\mathrm{TL}(\mathrm{dB})$ of roof & 17 & 22 & 30 \\
& $\mathrm{TL}(\mathrm{dB})$ of sidewall & 24.7 & 28.9 & 34.9 \\
\hline \multirow{2}{*}{ Case 2 } & $\mathrm{TL}(\mathrm{dB})$ of roof & 26 & 33 & 37 \\
& $\mathrm{TL}(\mathrm{dB})$ of sidewall & 24.7 & 28.9 & 34.9 \\
\hline
\end{tabular}




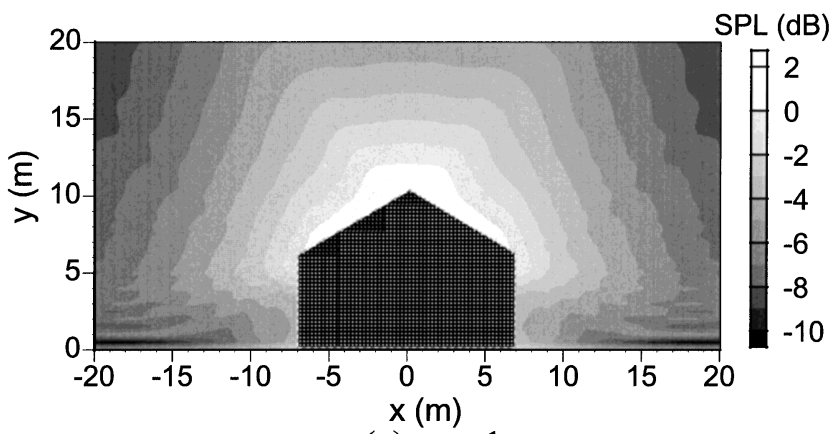

(a) case 1

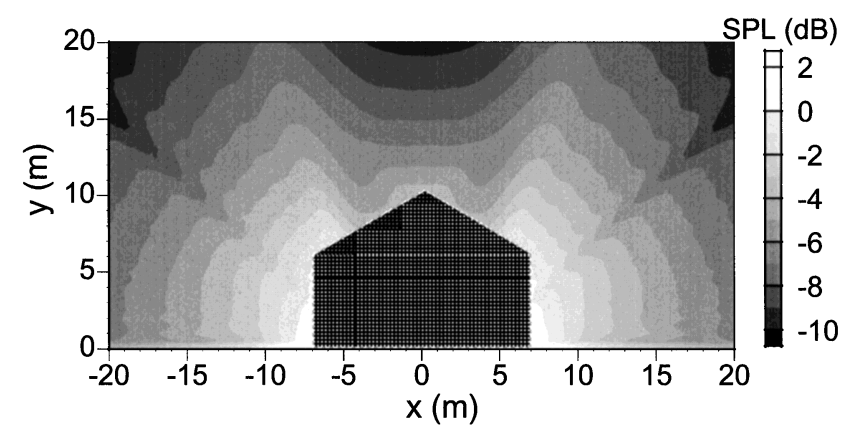

(b) case 2

Fig. 4 Distributions of sound pressure levels of a crosssection of the factory building at $500 \mathrm{~Hz}$.

a large sound source, such as a factory building, is not suitable for a comparison of patterns of directivity because the center position of the source can not be identified.

We then investigated the vertical profile of the sound pressure level. Calculations were made at $x=10$. Figures 5 and 6 show the results for case 1 and case 2, respectively. Changes in the sound pressure levels in the neighborhood of the ground surface were complicated due to interference between the direct, reflected, and diffracted waves. In case 1 (Fig. 5), where the transmission loss of the sidewall were larger than that of the roof, the sound pressure level was

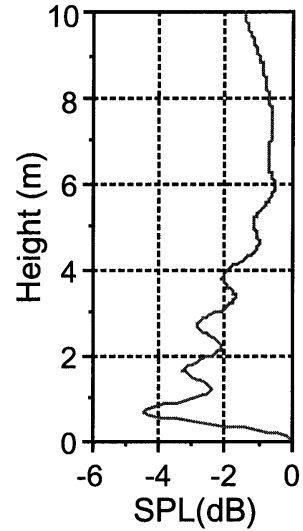

a) $250 \mathrm{~Hz}$

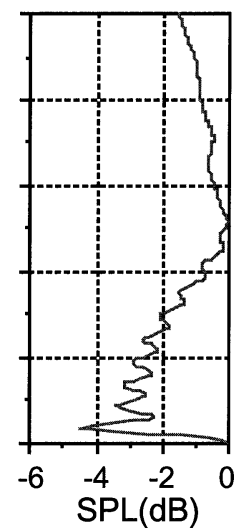

b) $500 \mathrm{~Hz}$

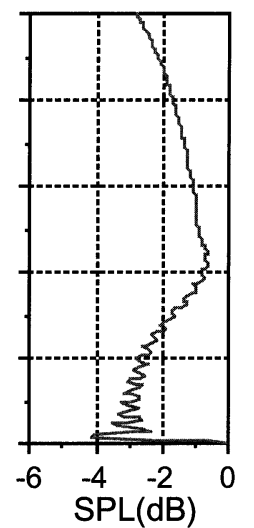

c) $1000 \mathrm{~Hz}$
Fig. 5 Vertical profiles of sound pressure levels at $x=10$ for case 1 .

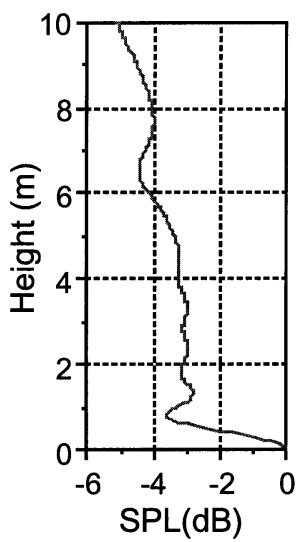

a) $250 \mathrm{~Hz}$

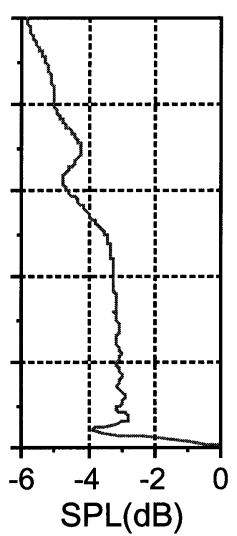

b) $500 \mathrm{~Hz}$

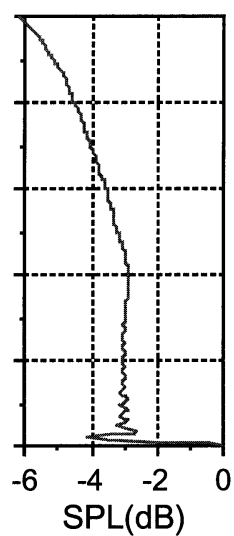

c) $1000 \mathrm{~Hz}$
Fig. 6 Vertical profiles of sound pressure levels at $x=10$ for case 2 .

maximum at a height of between $3 \mathrm{~m}$ and $7 \mathrm{~m}$ for frequencies between $250 \mathrm{~Hz}$ and $1,000 \mathrm{~Hz}$. Further, the sound pressure level of such a maximal position was between $2 \mathrm{~dB}$ and $3 \mathrm{~dB}$ larger than that in the neighborhood of the ground surface. In case 2 (Fig. 6), where the transmission loss of the sidewall was smaller than that of the roof, the sound pressure level was nearly constant up to a height of about $4 \mathrm{~m}$. This might be a consequence of the efficiency of radiation from the sidewall, i.e., a surface $5 \mathrm{~m}$ in height and of infinite length.

\section{Conclusion}

In order to investigate the sound field around a factory building we proposed a numerical model assuming a diffuse sound field inside the building and we evaluated the radiated sound from the sidewalls and roof using the geometrical theory of diffraction. The calculated values showed good agreement with the values measured from a scale model. The calculated values showed that the center position considering radiation all over the surface of the building could move up and down according to the pattern of transmission loss of the building materials. Furthermore, an analysis of the vertical profile of the sound pressure level indicated that the sound pressure levels changed little when the transmission loss of the roof was larger than that of the sidewall. Nevertheless, in the case of a normal combination of building materials, i.e., when transmission loss of the sidewall is larger than that of the roof, the sound pressure level would be distinctly maximum above the ground.

\section{References}

[1] G. Matsumoto, K. Fujiwara and A. Omoto, "A study on the insertion loss of a noise barrier for a directional sound source", J. Acoust. Soc. Jpn. (E), 20, 325 (1999).

[2] J. B. Keller, "Diffraction by an aperture", J. Appl. Phys., 28, 426 (1957).

[3] T. Kawai, "Sound diffraction by a many sided barrier or pillar", J. Acoust. Soc. Jpn. (J), 34, 369 (1978).

[4] Architectual Institute of Japan, Guideline in Practical Noise Control (Gihodo, Tokyo, 1994), p. 121. 